LIAMES 3 - pp.97-110, Primavera 2003

Ana Gerzenstein

(CONICET/UBA)

Beatriz Gualdieri

(Universidad Nacional de Luján - Argentina)

\title{
La armonía vocálica en lenguas chaqueñas de las familias Guaycurú y Mataguaya
}

\begin{abstract}
The paper deals with the harmony processes of vowels in aboriginal languages spoken in the region of the Chaco, located in the countries of Argentina and Paraguay. Specifically, the languages involved in this study are maká and chorote of the Mataguayan family, and mocoví, pilagá and toba members of the Guaycuruan family. There are identified and then described different types of harmony processes in each of these languages. Furthermore, it is presented a brief interpretation of these processes within the theory of autosegmental phonology. The results of the analysis will be relevant for all of those interested in diachronic and comparative studies of these languages, as well as for the current theories in phonology. KEYWORDS: Languages of Argentinean and Paraguayan Chaco; Vowel Harmony; Autosegmental Phonology; Guaycuruan and Mataguayan Families.
\end{abstract}

\section{RESUMEN}

Se analiza el proceso fonológico de armonía vocálica en lenguas aborígenes chaqueñas de las familias Mataguaya (lenguas maká y chorote 1 y 2) y Guaycurú (lenguas mocoví, pilagá y toba), habladas en el Chaco argentino y paraguayo. Son identificados y descritos los diferentes tipos de armonía vocálica presentes en cada una de estas lenguas, entendiendo por "armonía vocálica" el proceso fonológico por el cual las vocales, en un dominio específico (típicamente la palabra), armonizan en un rasgo dado. Asimismo, reexpone una interpretación preliminar de dicho proceso dentro del marco de las teorías fonológicas contemporáneas, específicamente de la corriente Autosegmental. El análisis que se presenta aportará, seguramente, información sistematizada sobre este fenómeno, tanto para estudios de tipo diacrónico que buscan establecer relaciones genéticas entre lenguas, como para la perspectiva tipológica y los modelos fonológicos actuales.

PALABRAS-CLAVE: Lenguas del Chaco Argentino-Paraguayo; Armonía Vocálica; Fonología Autosegmental; Familias Guaycurú y Mataguaya. 


\section{INTRODUCCIÓN}

En el presente trabajo examinaremos el fenómeno fonológico de la armonía vocálica $(\mathrm{AV})^{1}$ en cinco lenguas indígenas chaqueñas pertenecientes a las familias lingüísticas mataguaya (maká y chorote en sus dos variedades) ${ }^{2}$ y guaycurú (mocoví, toba y pilagá). Aunque con diferentes grados de vitalidad, todas ellas se hablan actualmente en la Argentina, excepto el maká cuyos hablantes viven en el Paraguay.

El trabajo se propone exponer datos ignorados o poco conocidos sobre la $\mathrm{AV}$ en estas lenguas. El análisis que presentamos aportará, seguramente, información sistematizada sobre este fenómeno que puede ser utilizada tanto para estudios de tipo diacrónico, que buscan establecer relaciones genéticas entre las lenguas, como para las perspectivas tipológica y teórica.

Las familias lingüísticas mataguaya y guaycurú agrupan la mayor parte de las lenguas de la región chaqueña. ${ }^{3}$ La primera está constituida por wichí (mataco), chorote, maká y niwaklé (chulupí) y la segunda, por kadiweu, mocoví, pilagá y toba. ${ }^{4}$

En diversas oportunidades se discutió la posibilidad de que existiera una relación genética entre las lenguas pertenecientes a estas dos familias y las últimas investigaciones indican que el parentesco entre ambas es altamente probable (Viegas Barros, 1993; 2003).

A los efectos del presente trabajo, la mayor parte de los datos empíricos que analizamos han sido recogidos directamente por las autoras, en sucesivas investigaciones de campo realizadas con hablantes que tienen como primera lengua, la indígena. Esto es así en el caso de ambas variedades de chorote, maká y mocoví. Los datos de pilagá y toba, por otro lado, provienen de fuentes indirectas (Messineo, 2000; Vidal, 2001).

A continuación, presentamos los resultados de nuestra investigación. En una primera sección, caracterizamos el fenómeno que estudiamos. A continuación se presenta una descripción de la $\mathrm{AV}$ en ambas familias linguísticas comenzando por las lenguas guaycurúes,

${ }^{1} \mathrm{El}$ análisis de los datos de las lenguas mataguayas es de la autoría de Gerzenstein; el de las lenguas guaycurúes de Gualdieri.

${ }^{2}$ No hemos considerado en nuestro estudio el wichí (mataco) porque la información bibliográfica con la que contamos no menciona el fenómeno de la armonía vocálica en esa lengua. Por otra parte, las investigadoras que actualmente están trabajando en tal lengua (Vidal, Terraza y Gerzenstein) todavía no han encontrado los datos empíricos pertinentes que les permitan abordar el tema.

${ }^{3}$ La región chaqueña, hábitat originario de los grupos Guaycurú y Mataguayo, es una vasta llanura con porciones boscosas que se extiende desde el planalto brasileño, por el norte: los ríos Paraná y Paraguay, por el este: el río Salado, por el sur, y los primeros contrafuertes andinos, por el oeste (Martínez Sarasola, 1992: 43).

${ }^{4}$ El pueblo maká (alrededor de 1.000 personas) es oriundo del Chaco paraguayo, pero fue relocalizado y actualmente vive en Mariano Roque Alonso, a 30 kilómetros de la ciudad de Asunción (Paraguay). Los hablantes de chorote 1 vivían, en el momento de la recolección de los datos, en Misión La Paz y en Tartagal (ambas localidades en la provincia de Salta) y los hablantes de chorote 2, en Filadelfia y Santa Rosa (Chaco paraguayo). El pueblo mocoví, con una población de entre 5.000 y 7.000 personas, se asienta en las regiones sur de la provincia del Chaco y norte y centro de la provincia de Santa Fe. Los tobas, por otra parte, ocupan vastos territorios en las provincias de Formosa, Chaco y este de Salta, así como asentamientos semiurbanos en las provincias de Santa Fe y Buenos Aires. Se trata de uno de los pueblos originarios más numeroso del país: entre 36.000 y 60.000 personas (Censabella, 1999). Los pilagás habitan en la provincia de Formosa y son aproximadamente 4.000 personas. 
donde este proceso está más extendido, y continuando con las lenguas mataguayas. Para finalizar presentamos una sistematización comparativa de los fenómenos analizados.

\section{LAARMONÍA VOCÁLICA}

La armonía vocálica (AV) es un fenómeno fonológico por el cual las vocales, en un dominio dado (típicamente la palabra), concuerdan en un rasgo fonológico particular. En nuestro trabajo consideraremos la AV desde una perspectiva amplia, incluyendo también aquellos procesos locales, como metafonía o umlaut, que afectan solamente a una sílaba a la derecha o a la izquierda.

La AV toma en consideración sólo las vocales ignorando, normalmente, las consonantes; sin embargo, las mismas pueden producir algún efecto bloqueando, por ejemplo, la interacción entre vocales (van der Hulst \& van de Weijer, 1995: 526-529).

En el estudio de la AV se distingue entre segmentos "opacos" y segmentos "transparentes". Los primeros bloquean la propagación del proceso y pueden, adicionalmente, determinar un dominio por sí mismos. Los segundos, por el contrario, son "invisibles" para los procesos de armonía, lo que quiere decir que permiten la propagación de los rasgos.

La AV se evidencia cuando operan procesos morfológicos de afijación y las vocales de la raíz y las de los afijos comparten algún rasgo. Generalmente, la AV es controlada por la raíz; es decir que con mayor frecuencia, se observa que los afijos alternan regularmente para cierto rasgo armonizando, en cuanto al mismo, con la raíz. Sin embargo, se registran casos en que la AV puede ser controlada por los afijos, afectando así a la raíz: es lo que sucede en las lenguas guaycurúes y mataguayas, como se verá más adelante.

Otro aspecto que permite caracterizar la $\mathrm{AV}$ es la dirección en que opera el proceso. Así pueden distinguirse dos tipos de AV: los casos en que el rasgo pertinente se propaga en una sola dirección, de derecha a izquierda o de izquierda a derecha, y aquéllos en que el rasgo pertinente se propaga en ambas direcciones.

\section{DESCRIPCIÓNDE LA AV ENLENGUAS CHAQUEÑAS}

\subsection{Lenguas guaycurúes (mocoví, toba, pilagá) ${ }^{5}$}

El análisis se centrará en la lengua mocoví, ${ }^{6}$ por razones de accesibilidad a los datos pertinentes (recogidos personalmente), haciendo referencia a aquéllos de las otras lenguas de la familia que permitan tipificar el proceso.

${ }^{5}$ Las abreviaturas usadas en este texto son: cho $=$ chorote (ambas variedades); cho1 = chorote

variedad $1 ; \mathbf{c h o 2}=$ chorote variedad $2 ; \mathbf{m a}=$ maká; $\mathbf{m o}=$ mocoví; $\mathbf{p i}=$ pilagá $;$ to $=$ toba .

${ }^{6}$ Los datos de mocoví corresponden a la variedad hablada en la provincia de Chaco (Gualdieri, 1998). 
En las lenguas guaycurúes, la AV involucra los rasgos fonológicos [alto] y [redondeado] y es desencadenada por procesos morfológicos tanto de prefijación como de sufijación. Incluiremos en nuestro análisis también ciertos procesos asimilatorios locales relacionados que son pertinentes para una comprensión más cabal de estos fenómenos.

\section{a) En contextos de sufijación}

Las lenguas guaycurúes presentan una morfología rica, especialmente en lo que respecta a sufijos, lo que determina, por lo tanto, la existencia de variados contextos morfológicos donde opera la AV.

a) 1. En lo que respecta a la AV de [alto], la vocal [i] de un sufijo desencadena $A V$ en las vocales anteriores de la base. Así, se propaga el rasgo de altura sobre todas las vocales coronales en la palabra, en dirección de derecha a izquierda. De esta manera, la oposición entre /e/ y /i/ se neutraliza. Las vocales no anteriores actúan como segmentos opacos, bloqueando la armonía, mientras que las consonantes actúan, en todos los casos, como segmentos transparentes: no importa la cualidad de las consonantes que se interponen entre las vocales, la modificación en altura vocálica sólo se detiene al encontrar una [a] o [o]. De hecho, parece ser el único caso, en las lenguas analizadas, de armonía vocálica en un sentido estricto, involucrando todas las vocales (excepto aquellas opacas, es decir [a] y [o]) de la palabra.

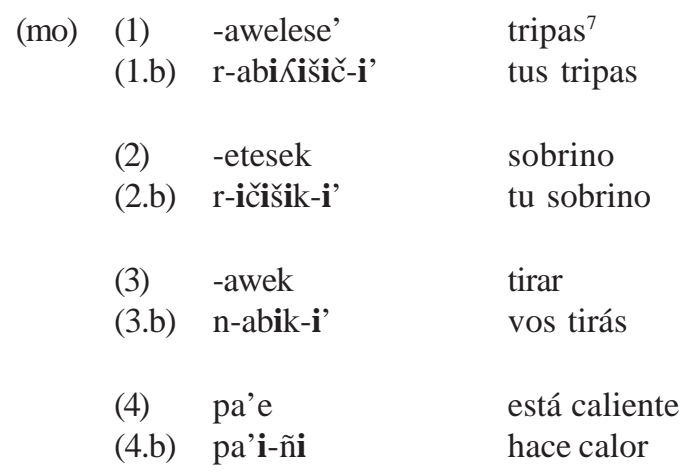

\footnotetext{
${ }^{7}$ Consignamos los símbolos utilizados que no se corresponden con los usuales en fonología: $\mathbf{b}=$ consonante labial continua sonora; $\mathbf{I}=$ vocal anterior alta abierta; $\mathbf{f} \mathbf{w}=$ consonante labial fricativa labializada; $\mathbf{L}=$ consonante lateral fricativa sorda; ' $=$ consonante laríngea oclusiva.

${ }^{8}$ La palatalización de coronales es un proceso productivo en las lenguas Guaycurú que involucra la asimilación regresiva de los rasgos de altura de [i] / [y] por las consonantes alveolares, produciendo segmentos alveopalatales. En mocoví y toba, se palatalizan todas las alveolares excepto la tap; en pilagá, solamente las alveolares oclusiva sorda, nasal y lateral. Relacionado con este proceso de palatalización se observa la modificación de la glide labial que, en el mismo contexto fonético, se realiza como consonante labial continua (Gualdieri, 1991).
} 
Nótese, en los ejemplos 1 y 2 , que este proceso proporciona el contexto necesario para la palatalización de las consonantes coronales. ${ }^{8}$

En toba ${ }^{9}$ y pilagá se observa el mismo comportamiento:

(to) (5) -emek

cuchara

(5.a) qor-imik-i

la cuchara de ustedes

(6) -wete

nuera

(6.a) qar-wiči-i

la nuera de ustedes

(pi) (7) -ede

escribir

(7.a) s-idi-ñi

yo escribo

a) 2. Con respecto a la $\mathbf{A V}$ de [redondeado] ${ }^{10}$, la presencia de una vocal redondeada [o] en la raíz produce la propagación, hacia el sufijo, de su característica labial sobre otra vocal posterior adyacente, de izquierda a derecha, siempre que la consonante que se interponga sea uvular, la que se comporta como segmento transparente. ${ }^{11}$ El resto de las consonantes bloquean la armonía.

\begin{tabular}{|c|c|c|c|}
\hline (mo) & $\begin{array}{l}(8) \\
(8 . b)\end{array}$ & $\begin{array}{l}\text { y-oča-qačit } \\
\text { ro-'o-qočit }\end{array}$ & $\begin{array}{l}\text { él/ella lo/la hace engordar } \\
\text { él/ella lo/la hace enojar }\end{array}$ \\
\hline & $\begin{array}{l}(9) \\
(9 . b)\end{array}$ & $\begin{array}{l}\text { r-alawat-aGan } \\
\text { kivo-Gon }\end{array}$ & $\begin{array}{l}\text { él/ella mata } \\
\text { él/ella lava (intr.) }\end{array}$ \\
\hline & $\begin{array}{l}(10) \\
(10 . b)\end{array}$ & $\begin{array}{l}\text { 1-qopi-aGa } \\
\text { n-poq-oGo }\end{array}$ & $\begin{array}{l}\text { la herida, el golpe } \\
\text { vergüenza }\end{array}$ \\
\hline (to) & $\begin{array}{l}(11) \\
(11 . b)\end{array}$ & $\begin{array}{l}\text { si-yo-Gon } \\
\text { la-žilo-Go }\end{array}$ & $\begin{array}{l}\text { yo lavo } \\
\text { su enojo, furia }\end{array}$ \\
\hline
\end{tabular}

La armonía transgutural afecta, también, a las vocales epentéticas, necesarias para cumplir con la silabificación correcta. Por un lado, en 12, 13 y 14 una restricción fonotáctica, que impide la coocurrencia de uvular+vocal coronal en la misma sílaba, desencadena la epéntesis de una vocal posterior, que copia las propiedades de la vocal de la sílaba precedente. La vocal epentética cumple función de núcleo silábico y separa la secuencia malformada. Ejemplos (cf. también 22.a):

${ }^{9}$ Messineo (2000: 62-3) interpreta estos datos como "palatalización vocálica".

${ }^{10}$ Tanto Messineo (2000: 57 ss) como Vidal (2001: 60 ss) interpretan el proceso como copia de todos los rasgos. En mi análisis, la vocal por defecto, en contexto de /q/ y /G/, es /a/ y el rasgo activo en la armonía es [redondeado].

${ }^{11}$ Las uvulares, faríngeas y laríngeas muestran, recurrentemente en las lenguas, características de segmentos transparentes en los procesos de armonía vocálica, denominados "armonía transgutural" (Rose, 1996). 
102

(mo) (12) n-emaG-ai:

(12.b) n-atoG-oi:

(to) (13) qaw-alaq-ay

(13.b) qo'-oq-oy

(pi) (14) awalaq-ae
LIAMES 3

la izquierda de ustedes

ustedes escupen

ustedes obedecen

la comida de ustedes

ustedes gritan

Por otro lado, en los ejemplos 15 y 16, la epéntesis vocálica es necesaria para una silabificación correcta: la vocal insertada ocupa, así, el núcleo silábico obligatorio. La vocal epentética es, normalmente, una coronal (15.b). La presencia de una uvular permite que opere la "armonía transgutural" desencadenada por la vocal [o] precedente:

(mo) (15) la'a:t-e'

(15.b) pioG-o'

(16) sapo-gi

(16.b) sapo-G-ogi

(to) (17) pioG-ol sus (pocas) casas

perros (pocos)

yo cierro

nosotros cerramos

perros (pocos)

En pilagá, Vidal (2001:46) menciona una restricción fonotáctica por la cual la faríngea (equivalente a [G] en mocoví y toba) ocurre siempre entre vocales idénticas /a/ o /o/. En mocoví, si bien al interior de raíces encontramos una restricción similar, existen casos en que la armonía no ocurre: por ejemplo, con los sufijos de Femenino [pioG-a] "perra" y de $1^{\text {a }}$ Persona Plural [ñatoG-aq] "nosotros escupimos", posiblemente debido a tratarse de vocales especificadas como /a/.

\section{b) En contextos de prefijación}

En los casos de prefijación, encontramos también armonía tanto de [alto] como de [redondeado]. La armonía afecta a la vocal del prefijo y es desencadenada por la primera vocal de la raíz.

b) 1. La AV de [alto], como en el caso de la sufijación, opera de derecha a izquierda, desencadenada por una vocal /i/ en la raíz y afectando al prefijo. Cualquier tipo de consonante funciona como segmento transparente. Las vocales epentéticas, necesarias para cumplir con los requerimientos de estructura silábica en contextos de prefijación, adquieren, normalmente, las características de altura correspondientes a la vocal inicial de la raíz y, a diferencia de lo que sucede en la sufijación, no se constituyen en contexto para la palatalización de alveolares, como se observa en los siguientes ejemplos:

(mo) (18) li-či

(18.a) ni-pia'

(18.b) ni-mik su pierna

$$
\text { [nIpia' nepia'] pie (de alguien) }
$$$$
\text { [nImik } \sim \text { nemik] nariz (de alguien) }
$$ 
En este caso parece tratarse de un proceso de características estrictamente alofónicas, opcional como lo indica la alternancia entre vocales de un continuo en grado de apertura: $[\mathrm{i}] \sim[\mathrm{I}] \sim[\mathrm{e}]$.

Algo similar acontece en toba, donde se observa opcionalidad en cuanto a la ocurrencia de la vocal alta epentética:
(19) si-wi'
(19.a) li-mik $\sim$ [lmik]
yo vengo
la nariz de él/ella
riñón (de alguien)

(19.b) ni-čigiši $\sim$ [nčigiši $]$

(pi) (20) li-kiyokena

su tobillo

(20.a) ni-piyosek

intestinos (de alguien)

Un proceso vinculado, que involucra la armonía en [alto], ocurre cuando un prefijo está constituido por un segmento palatal (glide [y] o consonante alveopalatal) y se inserta una vocal epentética, la que asume las características de altura de la consonante. ${ }^{12}$ Nótese que la vocal por defecto es [e] (ej. 22):

(mo) (21) -lew

(21.a) ži-lew

(22) se-naq

(22.a) ži-naq-ai’

(to) (23) ¿̌i-lew

(23.a) ži-waGan morirse

yo me muero

yo tiro (algo)

vos me tirás (algo)

yo me muero

él/ella me golpea

(pi) (24) ñi-tonaGak yo estoy feliz

Se trata de un proceso asimilatorio local ya que [alto] no se propaga indefinidamente a la derecha: las vocales coronales de la sílaba siguiente no modifican sus características articulatorias (cf. 22.b, 24.a).

b) 2. Con respecto a la $\mathbf{A V}$ de [redondeado], en casos de prefijación encontramos un comportamiento similar al que ocurre en los contextos de sufijación: vocales epentéticas copian los rasgos de una vocal [o] parte de la raíz, siempre que la consonante intermedia sea una uvular. El rasgo armónico es [redondeado]. Como ya se mencionó, las uvulares sólo pueden estar adyacentes a vocales que sean posteriores, razón por la cual toda vocal epentética en tal contexto sólo puede ser [a] o [o].

${ }^{12}$ Cabe mencionar que los prefijos constituidos por una consonante alveopalatal son interpretados como producto de la fusión de un segmento anterior alto, típicamente /i/, más una consonante alveolar: en los ejemplos 22.b, 23.a y 24, el prefijo /z/ </i+d/ (Messineo, 2000; Gualdieri, 1998). 
104

(mo) (25) ña-qa'Ganñi

(25.a) žo-qopat

(25.b) ño-qo'Gon

(25.c) no-qo'Gon [nqo'Gon]
LIAMES 3

yo me siento

yo tengo hambre

yo orino

él/ella orina

Nótese que, cuando el prefijo puede ser silabificado como núcleo (en la $3^{\mathrm{a}}$ persona), la epéntesis vocálica es opcional.

\begin{tabular}{|c|c|c|}
\hline (to) & $\begin{array}{l}\text { (26) da-qanaGan } \\
\text { (26.a) so-qolget } \\
\text { (26.b) ño-qoepa }\end{array}$ & $\begin{array}{l}\text { él/ella destruye } \\
\text { yo dudo } \\
\text { mi ceja }\end{array}$ \\
\hline (pi) & $\begin{array}{l}\text { (27) na-qaepa } \\
(27 . a) \text { no-qote }\end{array}$ & $\begin{array}{l}\text { ceja (de alguien) } \\
\text { su codo }\end{array}$ \\
\hline
\end{tabular}

Lo mismo ocurre cuando el segmento intermedio es una laríngea: ${ }^{13}$

(mo) (28) ne-'et él/ella bebe

(28.a) so-'o yo me enojo

\subsection{Lenguas mataguayas (chorote1, chorote2, maká)}

En las lenguas mataguayas, la AV involucra el rasgo fonológico [alto] y la copia de todos los rasgos. Se desencadena cuando ocurren procesos morfológicos de prefijación y/ o sufijación.

\section{a) En contextos de prefijación}

a) 1. En la AV de [alto], el factor desencadenante es la vocal / i / o la glide / y /. El elemento afectado es la primera vocal de la raíz verbal. El resultado es el desplazamiento de esta vocal y, / o la diptongación con / y /, de acuerdo con las siguientes especificaciones:
a $>$ e / ya, ye
e $>\mathrm{i}$
$\mathrm{o}>\mathrm{u} / \mathrm{yo}$
$\mathrm{u}>\mathrm{yu}$

La dirección del proceso es del prefijo a la raíz; es decir, de izquierda a derecha. Ejemplos:

${ }^{13}$ Según las propuestas de la fonología no lineal, las laríngeas carecen de rasgos de punto de articulación oral, por lo tanto no bloquean la propagación de tal tipo de rasgos. 


$\begin{array}{llll}\text { (cho) } & (29) & \text {-tsatit } & \text { mojar } \\ & (29 . a) & \text { a-tsatit } & \text { yo mojo } \\ (29 . b) & \text { i-tsyatit } & \text { él/ella moja } \\ (29 . c) & \text { i-tsyetit } & \text { él/ella moja } \\ & & \\ & & \text { yo amamanto } \\ (30) & \text { a-tepisye' } & \text { ella amamanta } \\ (30 . a) & \text { i-tipisye' } & \\ & & & \text { la barba } \\ \text { (cho) } & (31) & \text { im-poxsi' } & \text { tu barba } \\ & (31 . a) & \text { a-poxsi' } & \text { mi barba } \\ & (31 . b) & \text { i-puxsi' } & \\ & & \text { oreja } \\ \text { (ma) } & \text {-ekfi' } & \text { su oreja } \\ (32 . a) & \text { L-ekfi' } & \text { mi oreja } \\ (32 . b) & \text { y-ikfi' } & \text { nuestra oreja (incl.) }\end{array}$

(Para más ejemplos, véase Gerzenstein. 1978:61 ss y 1983:40-44)

En chorote 1 se han observado algunas restricciones específicas. Así, en las raíces que empiezan con un segmento coronal, la armonía opera exclusivamente si el prefijo está constituido sólo por la vocal /i/. En cambio, en chorote 2 y en maká, el desencadenante puede ser cualquier forma que contenga esa vocal. Ejemplos:

\begin{tabular}{|c|c|c|c|}
\hline (cho) & (33) & $-\mathbf{e}$ & lavar \\
\hline (cho1/2) & (33.a) & i-li & yo lavo \\
\hline (cho1) & (33.b) & si-le & lavamos \\
\hline (cho2) & (33.c) & si-li & lavamos \\
\hline (cho) & (34) & -sexlati' & sacar punta \\
\hline (cho1/2) & (34.a) & i-sixlati' & saco punta \\
\hline (cho1) & (34.b) & xi-selati' & él/ella saca punta \\
\hline (cho2) & (34.c) & xi-silati' & él/ella saca punta \\
\hline (cho) & $(35)$ & -na’ & padre \\
\hline (cho1/2) & (35.a) & i-nya' & mi padre \\
\hline (cho1) & (35.b) & si-na’ & nuestro padre \\
\hline (cho2) & (35.c) & si-nya' & nuestro padre \\
\hline \multirow[t]{5}{*}{ (ma) } & (36) & -ewheye' & esposo/a \\
\hline & (36.a) & L-ewheye' & su esposo/a \\
\hline & (36.b) & y-iwheye' & mi esposo/a \\
\hline & (36.c) & in-iwheye' & nuestro esposo/a \\
\hline & (36.d) & wit-iwheye' & el/la esposo/a \\
\hline
\end{tabular}


Por otro lado, también en chorote 1 , la presencia de una consonante velar $(/ \mathbf{k} / \mathrm{o} / \mathbf{x} /)$ impide la armonización de la vocal. De manera que, en esa variedad, la consonante velar constituye un "segmento opaco" porque bloquea la propagación del fenómeno. En chorote 2, por el contrario, todas las consonantes se comportan como "transparentes". Ejemplos:

$\begin{array}{llll} & \text { (cho1) } & & \text { (cho2) } \\ \text { (37) } & \text {-kaxlik } & \text { hígado } & \text {-kaxlek } \\ \text { (37.a) } & \text { i-kaxlik } & \text { mi hígado } & \text { i-kyaxlek } \\ & & & \\ \text { (38) } & \text {-kofwax } & \text { enemigo } & \text { kofwax } \\ \text { (38.a) } & \text { i-kofwax } & \text { mi enemigo } & \text { i-kyofwax }\end{array}$

Si bien la AV en estas lenguas afecta a un solo segmento y no se extiende a los demás, en unas pocas palabras del chorote 1 se advierte la extensión del fenómeno más allá de la primera vocal. Así, en raíces que presentan dos vocales sucesivas heterosilábicas con los mismos rasgos, la propagación de [alto] afecta a ambas.

Ejemplos:

(cho1)
(39) -péel
sombra
(39.a) i-píil
mi sombra
(40) tsa-xáan
soy sabio
(40.a) i-xéen
es sabio
(41) a-tóon
llevo
(41.a) xi-tyúun
él/ella lleva

a) 2. La copia de rasgos es otro proceso de AV, que (dentro de las lenguas que estamos examinando) afecta solamente al maká en contextos de prefijación. Consiste en la copia de todos los rasgos de la primera vocal de la raíz en los prefijos de $2^{\mathrm{a}}$. y $3^{\mathrm{a}}$. (a veces primera) personas. De esta manera, cuando la primera vocal de la raíz es / a /, la vocal del prefijo es / a /; cuando es / o/, la vocal es / o/. En el caso de las vocales / e, i, u /, la vocal del prefijo es / e /. El proceso se desencadena por la necesidad de una vocal entre la(s) consonante(s) del prefijo y la raíz que permita obtener una sílaba bien formada. En este proceso, el elemento afectado es el prefijo; el resultado es la copia de rasgos, y la dirección es desde la raíz al prefijo; es decir, de derecha a izquierda. Los ejemplos siguientes ilustran el proceso:

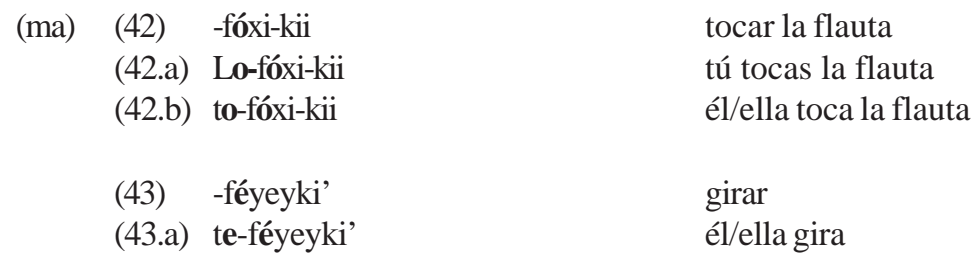




\begin{tabular}{|c|c|c|}
\hline (44) & -qaweX & garganta \\
\hline (44.a) & ya-qaweX & mi garganta \\
\hline (44.b) & a-qaweX & tu garganta \\
\hline (44.c) & La-qawex & su garganta \\
\hline (45) & ha-wanqa & yo me lavo las manos \\
\hline (45.a) & La-wanqa & tú te lavas las manos \\
\hline (46) & -noki’ & codo \\
\hline (46.a) & yi-noki' & mi codo \\
\hline (46.b) & o-noki' & tu codo \\
\hline (46.c) & Lo-noki' & su codo \\
\hline (46.d) & in-(n)oki' & nuestro codo \\
\hline (47) & Lewis & lindo \\
\hline (47.a) & ye-Lewis & soy lindo \\
\hline (47.b) & e-Lewis & eres lindo \\
\hline (47.c) & xe-Les-its & somos lindos/as \\
\hline (48) & -kumkenet & muslo \\
\hline (48.b) & yi-kumkenet & mi muslo \\
\hline (48.c) & e-kumkenet & tu muslo \\
\hline (48.d) & Le-kumkenet & su muslo \\
\hline
\end{tabular}

Cabe señalar que este proceso parece abarcar más de una sílaba en la palabra. Es el caso de los siguientes ejemplos, donde se observa armonía en todas las sílabas que preceden a la raíz, en estos casos las marcas de posesión no inherente y persona poseedora:

$\begin{array}{lll}\text { (ma) } & \text { nenek } & \text { cuchara } \\ \text { (49.a) } & \text { ye-qe-nenek } & \text { mi cuchara } \\ (49 . b) & \text { e-qe-nenek } & \text { tu cuchara } \\ (49 . c) & \text { Le-qe-nenek } & \text { su cuchara } \\ & & \\ (50) & \text { koyoyoy } & \text { carro } \\ (50 . a) & \text { yo-qo-koyoyoy } & \text { mi carro } \\ (50 . b) & \text { in-qo-koyoyoy } & \text { nuestro carro }\end{array}$

Cabe, asimismo, mencionar aquí la vinculación de la AV con la gramática en la lengua maká, que utiliza la copia de rasgos en los paradigmas nominales y verbales sólo con verbos de baja transitividad. Veamos los siguientes ejemplos:

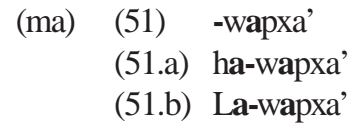

moverse

yo me muevo

tú te mueves 
Cuando la transitividad se aumenta (por un proceso de derivación para obtener el causativo, por ejemplo), se observa que los prefijos tienen siempre una vocal / i /:
(ma) (52) -wapxa-han
(52.a) hi-wapxa-han
hacer mover
(52.b) Li-wapxa-han
yo hago mover
(52.c) yi-wapxa-han
tú haces mover
el/ella hace mover

En el ejemplo que sigue, confróntese (53) con (54):
(ma) (53) -lixtsii
cantar
(53.a) hey-lixtsii
yo canto
(53.b) Le-lixtsii
tú cantas
(53.c) te-lixtsii
él/ella canta
(54) -lixtsi-nen
hacer cantar
(54.a) hi-lixtsinen
yo hago cantar
(54.b) Li-lixtsinen
tú haces cantar
(54.c) yi-lixtsinen
él/ella hace cantar

(Para más ejemplos, véase Gerzenstein, 2000:56 ss)

\section{b) En contextos de sufijación}

En contextos de sufijación, encontramos AV de [alto], igual que en la prefijación. El desencadenante es la vocal / i / o la glide / y /, que afectan a la última vocal de las raíces terminadas en / e /, a las cuales se sufija. El resultado es el desplazamiento de la altura de la última vocal de la base: / e / > / i /. La dirección del proceso es del sufijo a la raíz; es decir, de derecha a izquierda. Ejemplos:
(cho) (55) kinte'
(55.a) kinti-yis
diente
dientes
(56) axse'
(56.a) axsi-yis
hija
(57) aatse'
hijas
(57.a) t-axsi-tsi
pisar
pisan
(58) wole'
hoja
(58.a) woli-yis
hojas

(Para más ejemplos, véase Gerzenstein, 1978:64 y 1983:47) 


\section{CONCLUSIONES}

Comparando los datos expuestos, podemos decir que en las lenguas de ambas familias operan procesos de armonía vocálica. Tanto en las lenguas guaycurúes como en las mataguayas, la armonía de [alto] es desencadenada por una vocal [i]. Sin embargo, se observan importantes diferencias. En las lenguas guaycurúes el rasgo de altura se propaga a vocales coronales exclusivamente, produciendo un cambio /e/ > /i/. Toda consonante actúa como transparente y solamente las vocales posteriores bloquean el proceso. La dirección de la $\mathrm{AV}$ es siempre de derecha a izquierda: de los sufijos a la raíz y/o de la raíz a los prefijos. El dominio está constituido por la palabra, como se observa en los casos en que el elemento desencadenante es parte de un sufijo y propaga su especificación [alto] hacia todas las vocales coronales de la raíz. En los prefijos las fluctuaciones fonéticas [i] [I] [e] observadas en mocoví estarían indicando que el proceso de modificación en la altura vocálica opera en un nivel superficial (poslexical) de la derivación, razón por la cual las consonantes alveolares no están sujetas a palatalización (proceso lexical).

En las lenguas mataguayas, por otro lado, la armonía de [alto] afecta a todas las vocales del inventario fonológico y la modificación involucra sea un cambio en la altura de la articulación primaria, como en $/ \mathrm{a} />/ \mathrm{e} /, / \mathrm{e} />/ \mathrm{i} / \mathrm{y} / \mathrm{o} />/ \mathrm{u} /$, sea un diptongo constituido por la glide palatal, como en $/ \mathrm{a} />/ \mathrm{ya} / \sim / \mathrm{ye} /, / \mathrm{o} />/ \mathrm{yo} /, / \mathrm{u} />/ \mathrm{yu} /$. En chorote 1 las velares bloquean el proceso y las coronales también bloquean la armonía cuando el prefijo desencadenante tiene la forma CV. A diferencia de las guaycurúes, en estas lenguas el proceso es bidireccional y opera siempre controlado por los afijos afectando solamente a la primera sílaba adyacente de la raíz. En este sentido, la AV puede ser caracterizada como un proceso local, similar al denominado "metafonía" o "umlaut".

Un segundo caso de AV presente en las lenguas guaycurúes es el que involucra el rasgo [redondeado], que es desencadenado por [o] afectando a una vocal posterior. Se trata de la denominada "armonía transgutural", que opera exclusivamente cuando entre ambas vocales se interpone una uvular/faríngea (y laríngea, en mocoví), consonantes que actúan como segmentos transparentes. La AV, en este caso, actúa en forma bidireccional, controlada siempre por la raíz.

En la lengua mataguaya maká se documenta, también, un proceso de copia de rasgos de las vocales /a/ y /o/ de la primera sílaba de la raíz en los prefijos. La dirección, por tanto, es de derecha a izquierda y opera en el dominio de la palabra. Cabe mencionar que este tipo de AV se vincula con una distinción gramatical ya que opera en los prefijos personales y de posesión nominales y en los personales verbales solamente cuando se trata de verbos de baja transitividad, que están constituidos por una vocal no especificada. Con verbos de mayor transitividad, las marcas de persona contienen una vocal /i/.

Como se mencionó al inicio, esperamos haber presentado un análisis de un aspecto fonológico productivo en las lenguas guaycurúes y mataguayas que contribuya al desarrollo de distintas áreas de la lingüística, específicamente a los estudios histórico-comparativos, las propuestas tipológicas y las corrientes actuales de la teoría fonológica. 


\section{REFERENCIAS BIBLIOGRÁFICAS}

CENSABELLA, M. (1999). Las lenguas indígenas de la Argentina. Una mirada actual. Buenos Aires: EUDEBA.

GERZENSTEIN, A. (1978). Lengua chorote I. Buenos Aires: Instituto de Lingüística, Universidad de Buenos Aires.

.(1983). Lengua chorote. Variedad 2. Buenos Aires: Instituto de Lingüística, Universidad de Buenos Aires.

.(1984). Lengua maká. Estudio descriptivo. Buenos Aires: Instituto de Lingüística, Universidad de Buenos Aires.

.(2000). La transitividad en maká, una lengua indígena del Chaco paraguayo. In Luis Miranda (ed.) I Congreso de lenguas indígenas de Sudamérica, v. 1: 39-66. Lima, Perú: Universidad Ricardo Palma.

GUALDIERI, C.B. (1991). La palatalización en dos variedades mocovíes. In A. Gerzenstein (coord.). Temas de lingüística aborigen, p. 59-69. Buenos Aires Facultad de Filosofía y Letras, Universidad de Buenos Aires.

.(1998). Mocoví (Guaicuru). Fonologia e Morfossintaxe. Tesis de Doctorado. Campinas, SP: UNICAMP.

HULST, H. van der \& WIJER, J. van de (1995). Vowel Harmony. In J. Goldsmith (ed.). The Handbook of Phonological Theory, p. 495-534. Oxford-Cambridge: Blackwell.

MARTÍNEZ SARASOLA, C. (1992). Nuestros paisanos, los indios. Buenos Aires: Emecé.

MESSINEO, M.C. (2000). Estudio del toba hablado en la provincia del Chaco (Argentina). Aspectos gramaticales y discursivos. Tesis de Doctorado.Universidad de Buenos Aires (Argentina)

ROSE, S. (1996). Variable laryngeals and vowel lowering. Phonology 13: 73-117.

VIDAL, A. (2001). Pilagá grammar (Guaykuruan Family, Argentina). Ph. D. Dissertation. Oregon: University of Oregon.

VIEGAS BARROS, P. (1993). ¿Existe una relación genética entre las lenguas mataguayas y guaycurúes? In J. Braunstein (ed.). Hacia una nueva carta étnica del Gran Chaco V, p. 193-213. Informe de avance del CONICET. Las Lomitas, Formosa.

Recebido: 18/07/2003.

Aceito: 17/12/2003 